\section{Detection of early subclinical lung disease in children with cystic fibrosis by lung ventilation imaging with hyperpolarised gas MRI}

ABSTRACT Hyperpolarised ${ }^{3} \mathrm{He}$ ventilation$M R I$, anatomical lung MRI, lung clearance index ( $\mathrm{LCl})$, low-dose $\mathrm{CT}$ and spirometry were performed on 19 children (6-16 years) with clinically stable mild cystic fibrosis (CF) (FEV $>$ > -1.96), and 10 controls. All controls had normal spirometry, $\mathrm{MRI}$ and $\mathrm{LCl}$. VentilationMRI was the most sensitive method of detecting abnormalities, present in $89 \%$ of patients with $\mathrm{CF}$, compared with $\mathrm{CT}$ abnormalities in 68\%, $\mathrm{LCl} 47 \%$ and conventional MRI 22\%. Ventilation defects were present in the absence of CT abnormalities and in patients with normal physiology, including $\mathrm{LCl}$. Ventilation-MRI is thus feasible in young children, highly sensitive and provides additional information about lung structure-function relationships.

\section{BACKGROUND}

Lung ventilation heterogeneity due to the obstruction of small airways is an early and potentially reversible step in the progression of cystic fibrosis (CF). Individually, multiple breath washout (MBW), hyperpolarised (HP) gas ventilation-MRI and conventional structural lung imaging by CT have all been shown to be sensitive to early changes in the lungs before spirometry. ${ }^{1-3}$ The information they provide is complementary, and HP ventilation-MRI in particular has the potential to reveal airways obstruction and the functional consequences of regional structural changes detected with CT, as well as the nature of ventilation heterogeneity that prolongs gas washout in MBW. ${ }^{4}$ The aim of this study was to investigate the relative sensitivity of imaging and physiology assessments for the detection of early-stage lung disease in children with CF. In addition, we explored what insight functional ventilation imaging provides about the nature of ventilation abnormalities in the lungs in early $\mathrm{CF}$ and

Figure 1 Comparison of lung physiology and ventilation imaging metrics for healthy controls and patients with cystic fibrosis (CF). Each point represents a single subject; error bars represent group means and $95 \%$ $\mathrm{Cl}$ of the means. (A) FEV 1 z-score and (B) $\mathrm{FEV}_{1} / \mathrm{FVC} z$-score derived from spirometry. Horizontal dotted line indicates a z-score of zero. (C) Sitting lung clearance index $(\mathrm{LCl})$ and $(\mathrm{D})$ supine $\mathrm{LCl}$ from multiple breath washout. Horizontal dotted line represents upper limit of normal $\mathrm{LCl}$. (E) Unventilated lung volume percentage (UVP) and (F) mean coefficient of variation (CV) of ventilation imaging signal, from ${ }^{3} \mathrm{He}$ MRI.

\section{A}

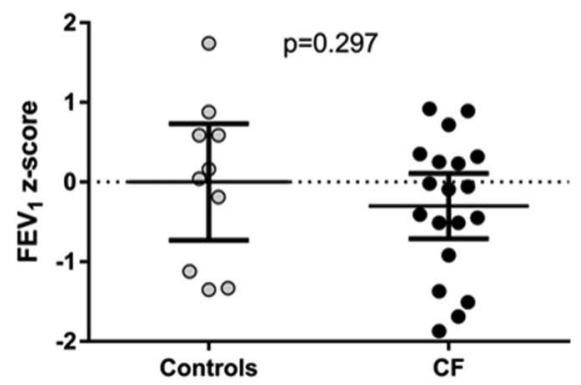

C

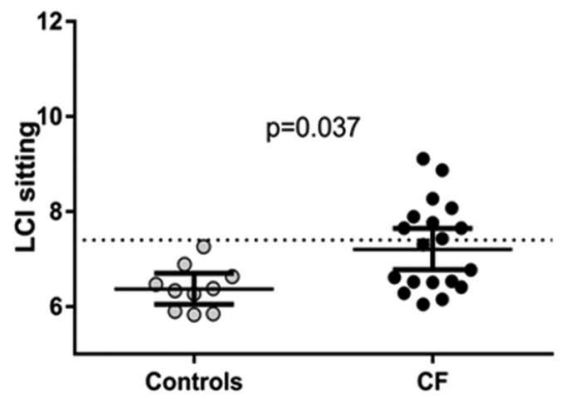

E

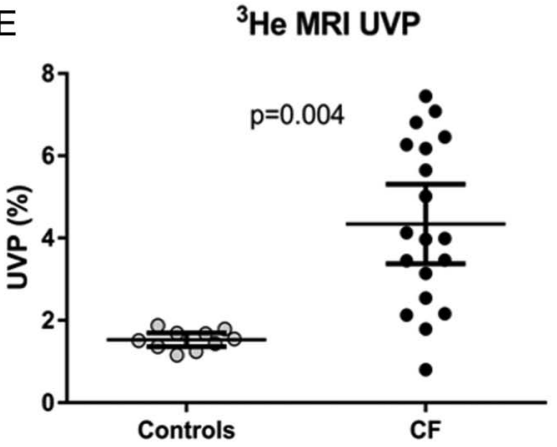

how these correlate with the more clinically scalable assessment of ventilation heterogeneity provided by MBW.

\section{METHODS}

For full technical details, see the online supplement.

Nineteen CF children and ten controls were assessed. Subjects attended on a single occasion, when clinically stable, and were assessed with $\mathrm{SF}_{6} \mathrm{MBW},{ }^{1}$ plethysmography, spirometry, $\mathrm{HP}{ }^{3} \mathrm{He} \mathrm{MRI}$ and ${ }^{1} \mathrm{H}$ MRI. Patients with $\mathrm{CF}$ also underwent inspiratory and expiratory chest $\mathrm{CT}^{5}$ All subjects had $\mathrm{FEV}_{1}$ z-score $>-1.96$ and were aged between 6 and 16 years old. To test for significant differences between the healthy control and $\mathrm{CF}$ patient groups, two-tailed paired tests were performed to reflect the age-matching of data. Sensitivity was defined as the presence of an abnormal measurement (ie, not within the normal range for that measurement) in the presence of the diagnosis of CF. This assumes that all patients should

\section{B $\quad$ FEV $_{1} /$ FVC $z$-score}

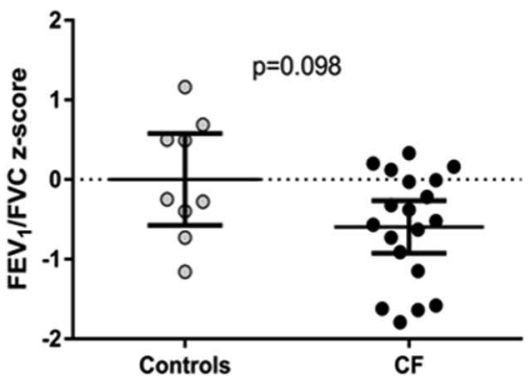

D

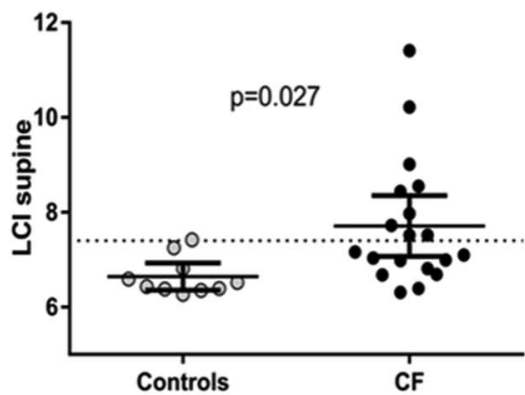

$\mathrm{F}$

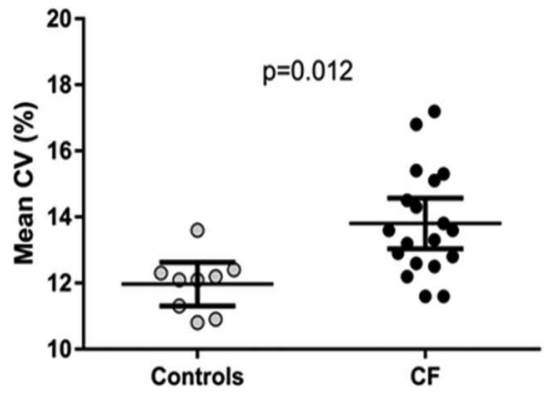


have some abnormality in at least one measurement, which may not be true in this population with very mild clinical expression of CF, and therefore provides a conservative estimation of sensitivity of the lung assessments. This study was approved by the National Research and Ethics Committee (REC 12/YH/0343) and parents/guardians provided written informed consent.

\section{RESULTS}

Full demographic and outcome data are presented in the online supplement. Patients with CF had early-stage lung disease, with no significant differences between CF and controls for any lung function measures except lung clearance index (LCI) (figure 1). In controls, no ventilation or structural abnormalities were detected on either ${ }^{3} \mathrm{He}$ or ${ }^{1} \mathrm{H}$ MRI (see online supplement). Both ${ }^{3} \mathrm{He}$ MRI quantitative measures were however significantly increased in patients with CF when compared with controls (figure 1). Ventilation heterogeneity and obstruction seen with ventilation-MRI generally corresponded to anatomical abnormalities detected by CT (see online supplementary figure S4). In several instances, however, ventilation defects were present on ventilation-MRI with no corresponding features of structural pathology detectable on CT (figure 2).

The presence of visible defects on ventilation-MRI had the greatest sensitivity for detecting evidence of $\mathrm{CF}$ airways disease: nine patients with $\mathrm{CF}$ (47\%) had elevated sitting LCI, when compared with $17 \quad(89 \%)$ with ventilation-MRI abnormalities. In contrast, structural abnormalities were detectable in four patients $(22 \%)$ with ${ }^{1} \mathrm{H}$ MRI and 13 patients (68\%) with CT. Ventilation-MRI detected abnormalities in four patients with normal CT; LCI was abnormal in only two of these. Two patients with mild variant disease (both R117H-7T heterozygotes) had no abnormality detected by any technique; these were the only patients with CF where ${ }^{3} \mathrm{He}$ MRI was normal.

A table of correlations is presented in the online supplement. LCI and ${ }^{3} \mathrm{He}$ MRI metrics showed significant moderate correlation and ${ }^{3} \mathrm{He}$ MRI \%-unventilated lung correlated significantly with CT Brody score and CT gas-trapping score. There was no significant correlation between LCI and CT scores.

\section{DISCUSSION}

This is the first study to combine the powerful functional technique of ventilation imaging using HP gas MRI with assessments of structural lung disease from CT and the whole lungs assessment of ventilation heterogeneity provided by MBW. Although both ${ }^{3} \mathrm{He}$ MRI and MBW measure aspects of ventilation heterogeneity in the lungs, the detailed spatial information provided by ventilation-MRI meant that abnormalities in ventilation distribution were visible even when these were insufficient to affect the LCI. Likewise, ventilation defects were visible in some cases in the absence of structural abnormalities on CT (eg, figure 2), which may be due to the inherent sensitivity of ventilation imaging to small airways obstruction that cannot be explicitly resolved on CT.

These are important findings for our understanding of how CF lung disease develops and how we interpret lung function data. In this cohort of patients with CF, specifically selected to represent those with the mildest airways disease, LCI was abnormal in almost half of all subjects $(47 \%)$, in keeping with prior observations. $^{1} 6$ Ventilation-MRI however was more sensitive than both LCI and CT and detected ventilation defects in all but two patients $(89 \%)$, both of whom had genetic variants that may not be associated with CF lung disease until adulthood. The great advantage of ventilation-MRI is that it offers detailed regional information about the nature and distribution of ventilation defects. Thus, in patients with early disease, we predominantly detected patchy ventilation defects distributed throughout the lung, with larger focal defects in areas where there was already evidence of structural damage on CT or ${ }^{1} \mathrm{H}$ MRI. In contrast, MBW represents a
Figure 2 Hyperpolarised gas MRI and CT imaging: examples of discordance in patients with cystic fibrosis (CF). Each row represents a single unique patient with $\mathrm{CF}$, with equivalent slices from hyperpolarised gas MRI, CT and ${ }^{1} \mathrm{H}$ MRI in the columns from left to right. In the first subject $(A-C),{ }^{3} \mathrm{He} M R I$ showed heterogeneous ventilation with widely distributed patchy, semiventilated defects (A). CT for this slice was normal (expiratory, shown in $\mathrm{B}$ ) and ${ }^{1} \mathrm{H}$ MRI (C) was normal throughout. In the second patient with $\mathrm{CF}$, small, subsegmental defects were observed throughout the lungs with ${ }^{3} \mathrm{He}$ MRI (D). CT for this slice was normal (expiratory, shown in E), but elsewhere showed lingular atelectasis and minimal air-trapping. ${ }^{1} \mathrm{H}$ MRI was normal for this slice (F) but showed lingular atelectasis elsewhere. In the final patient, several small ventilation defects were seen on ${ }^{3} \mathrm{He}$ MRI (G), while $\mathrm{CT}$ (inspiratory, shown in $\mathrm{H}$ ), ${ }^{1} \mathrm{H}$ MRI (I) and lung clearance index were all normal.

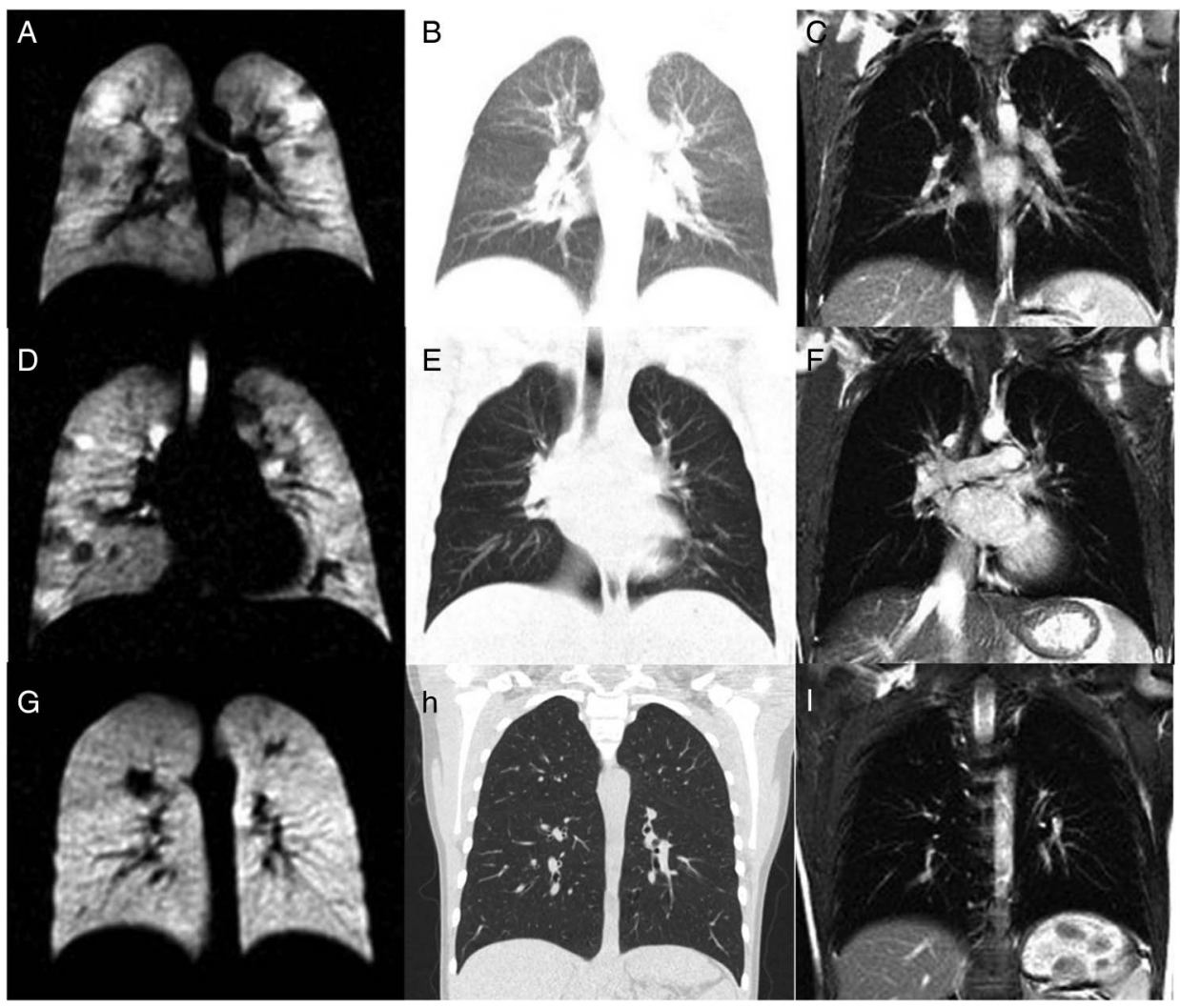


washout signal integrated over the whole lungs, so that mild ventilation heterogeneity can inevitably be masked, and differentiation of the signal into regional or anatomical lung compartments is at best speculative. $^{7}$

The ${ }^{1} \mathrm{H}$ MRI protocol deployed here was used to determine lung boundaries and was not optimised for structural imaging, where new methods such as 3D ultrashort echo time imaging should offer improvements. Alternative CT scoring systems and protocols also exist that may offer additional sensitivity. ${ }^{8}$ Finally, we did not perform gadolinium contrast-enhanced imaging, as we felt this would deter children from participating. Despite possible limitations in the ${ }^{1} \mathrm{H}$ MRI and CT protocol, it is clear that $\mathrm{HP}$ gas ventilation-MRI is a powerful tool for detecting early lung changes. The superior sensitivity and detail of information provided by ventilation-MRI also offers the prospect that this measurement will allow an earlier or more detailed radiation-free appreciation of the response to novel therapies than even LCI. ${ }^{9}$ The advent of high-quality HP gas ventilation-MRI using the cheaper and readily available ${ }^{129} \mathrm{Xe}$ isotope means that the technology is now much more readily clinically deployed. ${ }^{10}$ The ventilation-MRI presented here are thus exemplars of what may become routine assessments in detecting early disease and treatment effects.

In conclusion, in this population of children with CF with very mild lung disease, we have shown that ventilation-MRI is highly sensitive to detecting the consequences of airway disease. Even patients with apparently pristine lungs by all current physiology and imaging standards have evidence of ventilation-MRI abnormalities. HP gas MRI provides detailed regional information about disease severity and physiological impairment.

Helen Marshall, ${ }^{1}$ Alex Horsley, ${ }^{1,2}$ Chris J Taylor, ${ }^{3}$ Laurie Smith, ${ }^{1,3}$ David Hughes, ${ }^{3}$ Felix C Horn, ${ }^{1}$ Andrew J Swift, ${ }^{1}$ Juan Parra-Robles, ${ }^{1}$ Paul J Hughes, ${ }^{1}$ Graham Norquay, ${ }^{1}$ Neil J Stewart, ${ }^{1}$ Guilhem J Collier, ${ }^{1}$ Dawn Teare, ${ }^{4}$ Steve Cunningham, ${ }^{5}$ Ina Aldag, ${ }^{3}$ Jim M Wild ${ }^{1}$

Helen Marshall and Alex Horsley are joint first authors.
${ }^{1}$ POLARIS, Academic Radiology, University of Sheffield, Sheffield, UK

${ }^{2}$ Centre for Respiratory Medicine and Allergy, Institute of Inflammation and Repair, Manchester Academic Health Science Centre, The University of Manchester and University Hospital of South Manchester NHS

Foundation Trust, Manchester, UK

${ }^{3}$ Sheffield Children's Hospital, Sheffield, UK

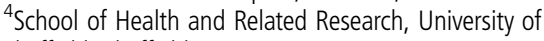
Sheffield, Sheffield, UK

${ }^{5}$ Department of Respiratory and Sleep Medicine, Royal Hospital for Sick Children, Edinburgh, UK

Correspondence to Professor Jim M Wild,

Department of Academic Radiology, Floor C, Royal

Hallamshire Hospital, Glossop Road, Sheffield S10 2JF, UK; j.m.wild@sheffield.ac.uk

Acknowledgements The authors are grateful to all the volunteers and their parents who took part in this research. The authors would like to personally thank Dr Noreen West, Dr Sonal Kansra and the CF team at Sheffield Children's Hospital, Leanne Armstrong for coordination of study visits and Karen O'Donnell for spirometry and plethysmography data acquisition in some subjects.

- Additional material is published online only. To view please visit the journal online (http://dx.doi.org/10. 1136/thoraxjn-2016-208948).

Contributors HM: MRI acquisition and analysis, overall data analysis, co-wrote the manuscript. AH: study design, MBW set-up and over-read of analysis, co-wrote the manuscript. CJT: study design, patient recruitment, reviewed the manuscript. LS: performed and analysed lung function and MBW measurements, reviewed the manuscript. DH: scored CT images and reviewed all MRI, reviewed the manuscript. FCH: performed MBW measurements, MRI acquisition, reviewed the manuscript. AJS: scored CT images, reviewed the manuscript. JP-R: polarised gas. PJH: MR image registration. GN, NJS and GJC: polarised gas, technical imaging support. DT: statistical support. SC: study design, reviewed the manuscript. IA: patient recruitment and consent, reviewed the manuscript. JMW: study design, MRI acquisition design, data analysis, co-wrote the manuscript, underwrites the work. The corresponding author had access to all the data in the study and accepts responsibility for its validity.

Funding This article presents independent research funded by the Cystic Fibrosis Trust and the National Institute of Health Research (NIHR)

Disclaimer The funders had no role in the study design, data collection, analysis, interpretation or preparation of this report. The views expressed are those of the authors and not necessarily those of the NHS, the NIHR or the Department of Health.

Competing interests None declared.

Patient consent Obtained.

Ethics approval National Research and Ethics Committee.
Provenance and peer review Not commissioned; externally peer reviewed.

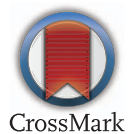

To cite Marshall H, Horsley A, Taylor CJ, et al Thorax 2017;72:760-762.

Received 22 May 2016

Revised 11 January 2017

Accepted 3 February 2017

Published Online First 6 March 2017

Thorax 2017;72:760-762.

doi:10.1136/thoraxjnl-2016-208948

\section{REFERENCES}

1 Horsley AR, Gustafsson PM, Macleod KA, et al. Lung clearance index is a sensitive, repeatable and practical measure of airways disease in adults with cystic fibrosis. Thorax 2008:63:135-40.

2 Bannier E, Cieslar K, Mosbah K, et al. Hyperpolarized ${ }^{3} \mathrm{He}$ MR for sensitive imaging of ventilation function and treatment efficiency in young cystic fibrosis patients with normal lung function. Radiology 2010;255:225-32.

3 de Jong PA, Nakano Y, Lequin $\mathrm{MH}$, et al. Progressive damage on high resolution computed tomography despite stable lung function in cystic fibrosis. Eur Respir J 2004:23:93-7.

4 Horsley A, Wild JM. Ventilation heterogeneity and the benefits and challenges of multiple breath washout testing in patients with cystic fibrosis. Paediatr Respir Rev 2015;16(Suppl 1):15-18.

5 Loeve $M$, Lequin $M H$, de Bruijne $M$, et al. Cystic fibrosis: are volumetric ultra-low-dose expiratory CT scans sufficient for monitoring related lung disease? Radiology 2009;253:223-9.

6 Gustafsson PM, Aurora P, Lindblad A. Evaluation of ventilation maldistribution as an early indicator of lung disease in children with cystic fibrosis. Eur Respir J 2003;22:972-9.

7 Horsley AR, Macleod KA, Robson AG, et al. Effects of cystic fibrosis lung disease on gas mixing indices derived from alveolar slope analysis. Respir Physiol Neurobiol 2008;162:197-203.

8 Rosenow T, Oudraad MC, Murray CP, et al., Australian Respiratory Early Surveillance Team for Cystic Fibrosis (AREST CF). PRAGMA-CF. A quantitative structural lung disease computed tomography outcome in young children with cystic fibrosis. Am J Respir Crit Care Med 2015;191:1158-65.

9 Davies J, Sheridan H, Bell N, et al. Assessment of clinical response to ivacaftor with lung clearance index in cystic fibrosis patients with a G551D-CFTR mutation and preserved spirometry: a randomised controlled trial. Lancet Respir Med 2013;1:630-8.

10 Stewart NJ, Norquay G, Griffiths PD, et al. Feasibility of human lung ventilation imaging using highly polarized naturally abundant xenon and optimized three-dimensional steady-state free precession. Magn Reson Med 2015;74:346-52. 